\title{
Radiation and Surface Roughness Effects in the Numerical Modelling of Enclosure Fires
}

\author{
S. KUMAR and G. COX* \\ Fire Research Station \\ Borehamwood Herts, WD6 2BL, UK
}

\section{ABSTRACT}

This paper concerns improvements to the three dimensional, time dependent, field model JASMINE to account for the effects of radiant heat transfer and of momentum and convective heat transfer to rough surfaces. Experimental fire tests conducted in a disused railway tunnel are compared with steady state predictions to examine the effects of these improvements. Attention is drawn to the lack of information on convective heat and momentum transfer to the surfaces of practical building

materials.

KEYWORDS: Mathematical Model, Field Model, Tunnel, Eire, Radiation, Rough Surfaces.

\section{INTRODUCTION}

At the previous Symposium, a reasonable degree of validation was demonstrated for the field model JASMINE when applied to three very different experimental configurations ${ }^{2}$. Attention was drawn, however, to the need to provide a more general treatment for the calculation of heat losses to the boundaries. It is the universality of the field modelling approach that is its strength, but experience had shown that it was necessary to adopt a lumped heat transfer coefficient approach to this problem ${ }^{2}$. Clearly a more general treatment is necessary if field models are to be genuinely universal in their applicability. This would need to account for radiative heat transfer and the effect of surface roughness on convective heat transfer, a factor that had become apparent to us in much earlier studies.

Although the calculation of radiative flux to isothermal surfaces and of convective heat transfer to smooth surfaces is fairly well established, application to non-isothermal, rough walls characteristic of internal building surfaces is much less so. This paper describes work to generalise these treatments using the six flux radiation model and a wall function treatment for rough surfaces to model convective heat flux and momentum transfer. It uses the earlier validation of JASMINE ${ }^{1},{ }^{3}$ against experimental fires conducted in a tumel as a vehicle for

*Author for all correspondence

(c) Crown copyright 1987 
comparison of the various treatments. The mathematical basis of the model has already been described in Refs $1-2$ and will not be repeated here.

\section{RADIATIVE HEAT TRANSFER}

For the accurate modelling of radiative heat transfer, particularly in the vicinity of the flaming source itself, proper consideration must be given to the detailed combustion products, their soot content and the effects of turbulent fluctuations in temperature and gas composition ${ }^{4}, 5$. This is particularly so very close to the source where attempts are being made to model flame spread over flammable surfaces. However, it is possible where concern is largely to estimate the effects of radiative transfer from the non-luminous products of combustion that have accumulated under a ceiling, to neglect these effects. Even with this simplification, the incorporation of radiative transfer in the field model is quite involved.

This is apparent from the nature of the radiant transfer equation. This can be obtained by considering the balance of monochromatic radiant energy of the pencil of rays passing in a specified direction $\vec{\Omega}$, through a small volume of participating medium. For example, for steady conditions and for coherent, isotropic scattering this equation is:

$\vec{\Omega} \cdot \vec{\nabla} I_{\lambda}(\vec{r}, \vec{\Omega})=-\left(a_{\lambda}+s_{\lambda}\right) I_{\lambda}(\vec{r}, \vec{\Omega})+a_{\lambda} I_{b, \lambda}(\vec{r})+\frac{s}{4 \pi} \lambda \int_{0}^{4 \pi} I_{\lambda}\left(\vec{r}, \vec{\Omega}^{\prime}\right) d \vec{\Omega}^{\prime}$

Where $I_{\lambda}(\vec{r}, \vec{b})$ is the monochromatio intensity of the radiant energy and is a function of wavelength, position and direction; $I_{b, \lambda}$ is the monochromatic intensity of black body radiation at the temperature $T$ of the medium and $a_{\lambda}$ and $s_{\lambda}$ are respectively the spectral absorption and scattering coefficients of the medium at the wavelength $\lambda$.

If the effects of turbulent fluctuations are ignored each variable takes on its time-mean value. Equation (1) forms the basis for the quantitative study of radiant energy transfer in a participating medium. Its solution is particularly difficult because of its integro-differential form but also because of the angular, spatial and spectral dependence of the radiant intensity. An excellent review of the various solution methods that can be used to resolve this problem, has recently been given by Viskanta and Menguc ${ }^{6}$.

In common with many other workers in the combustion field, a six-flux method has been used in this study because its differential form is compatable with that of the flow field conservation equations. Whilst reasonably acceptable for dealing with heat transfer from stratified layers the flux methods are not suitable for the prediction of radiant transfer to combustible fuels ahead of a spreading flame front. Here the discrete transfer method ${ }^{7}$ appears more promising.

The six-flux model assumes that the angular and spatial dependence of radiation intensity are separable and that intensity is uniform in given intervals of the solid angle. In what follows the dependence of properties of the gas on wavelength has been ignored here but this is not an essential feature of the model. If $\mathrm{E}_{i}^{+}$and $\mathrm{F}_{i}^{-}$represent forward and backward radiation fluxes passing through the control volume in the $i$ direction and $\mathrm{E}_{\mathrm{b}}$ is the total black body emissive power of the gas, then 
it can be shown that the generalised equation of the six-flux model is of the form

$\frac{d}{d x_{i}}\left[2 \Gamma_{R} \frac{d R_{i}}{d x_{i}}\right]+s_{i}=0, \Gamma_{R}=\frac{1}{a+s}$ where,

$$
\begin{aligned}
& S_{i}=\frac{d q_{i}^{\prime \prime}}{d x_{i}}=-2(a+s) R_{i}+2 a E_{b}+2 \frac{s}{3} \sum_{i=1}^{3} R_{i} \\
& R_{i}=\frac{1}{2}\left(F_{i}^{+}+F_{i}^{-}\right) \text {and } \dot{q}_{i}^{\prime \prime}=\left(F_{i}^{+}-F_{i}^{-}\right)
\end{aligned}
$$

This is of the same form as the generic conservation equation solved in JASMINE

$$
\frac{\partial}{\partial t}(\rho \Phi)+\operatorname{div}\left(\rho \vec{u} \Phi-\Gamma_{\Phi} \vec{\nabla} \Phi\right)=S_{\Phi}
$$

It is worth mentioning here that the average radiation flux, $R_{1}$, is introduced simply for mathematical convenience. It has no particular physical significance. The net radiation flux $\dot{q}_{i}^{\prime \prime}$ determines the proportion of the total enthalpy lost by radiation to the walls and openings of the compartment boundaries.

The source term of the radiation equation (2) is a sink term in the enthalpy equation (3) (ie when $\Phi=h$ ).

$s_{h}=-\sum_{i=1}^{3} s_{i}=2 a \sum_{i=1}^{3} R_{i}-6 a E_{b}$

At the walls, the heat flux lost from the gas phase will depend upon the wall emissivity $e_{W}$, reflectivity $r_{w}$ and its temperature $\mathrm{T}_{w}$. For wavelength independent surface properties this flux will equal its radiosity. Thus, if $\mathrm{F}_{j}^{+}$is the flux incident on a surface, the flux returning is

$F_{i}^{-}=\left(1-e_{w}\right) F_{i}^{+}+e_{w} E_{b, w}$

Thus the net radiation flux in the positive direction leaving the gas phase is

$\dot{q}_{i}^{\prime \prime}=\frac{2 e_{w}}{2-e_{w}}\left[R_{i}-E_{b, w}\right]$

At an opening, the net radiation flux leaving the enclosure is

$\ddot{q}_{i}^{\prime \prime}=2\left(R_{i}-e_{o p} \sigma T_{o p}^{4}\right)$

where $e_{o p}$ and $T_{o p}$ are the emissivity and temperature of the cell at the opening and 0 is the Stefan Boltzmann constant. Equations (5) and (6) 
are sink terms to the radiation equation at the wall and opening respectively.

MOMENTUM AND CONVECTIVE HEAT LOSS TO BOUNDARIES

Flow field calculations can be severely affected by the surface characteristios of the enclosure boundaries. Generally rough surfaces lead to an enhancement of heat and momentum transfer to the boundaries.

Most of the data that are available for practical surfaces relate to machined or turned metal. There have been few studies of building materials that are of interest not only to fire researchers but also to those involved in developing thermal models of buildings for energy conservation purposes.

The complexity of the subject arises because of the large variations in shapes, sizes and distribution of the roughness elements, as well as their coupling with the fluid stream. This coupling dictates how far the influence of roughness propagates into the fluid stream so as to affect the entrainment and dissipation processes, and in turn the heat and momentum transfer.

For the accurate calculation of wall fluxes it is very important that the structure of the boundary layer attached to the wall surface is well understood. This is because the flow of turbulent fluid over a solid boundary generates very steep velocity and temperature gradients near the solid surface. The usual treatment of this problem in all engineering field models is to bridge the region using the well known wall functions. For velocity and temperature respectively this implies the following relationships using conventional near wall scaling

$u_{+}=\frac{u_{P}}{u_{*}}=\frac{1}{k} \ln \left(E y_{+}\right) ; \quad T_{+}=\frac{T_{w}-T_{P}}{T_{*}}=\operatorname{Pr}\left(u_{+}+P_{j}\right)$

where $y_{+}=\frac{y_{P} u_{*}}{v}, u_{*}=\sqrt{\tau_{w} / \rho}, T_{*}=\dot{q}_{W}^{\prime \prime} / \rho C_{P} u_{*}$

Here $y_{\mathrm{p}}$ is the normal distance from the surface to the near wall node, $P$, placed in the fully turbulent region, $u_{*}$ and $T_{*}$ are respectively the friction velocity and friction temperature. $k$ is the von Karman constant, $P r_{t}$ is the turbulent Prandt 1 number, $\tau_{W}$ and $\ddot{q}_{w}$ are the wall shear stress and heat flux respectively. $\rho, v$ and $c_{p}$ are the density, kinematic viscosity and specific heat of the fluid and $T_{W}$ and $T_{p}$ are respectively the wall and fluid temperatures. $E$ and $P_{j}$ are constants for a particular surface but $E$ does vary sharply with the surface roughness whilst $P_{j}$ depends mainly on the molecular Prandt 1 number of the fluid, representing the extra resistance to heat transfer offered by the laminar sub layer. This treatment for smooth boundaries is well established. However for rough surfaces there is more uncertainty.

The universality of equation (7) for velocity in particular has been demonstrated for a wide range of flows in pipes and in boundary layers with smooth and rough surfaces ${ }^{8}$. The variation of $E$, as illustrated by Jayatilleke ${ }^{s}$, with roughness Reynolds number, $R_{r}=y_{r} u_{*} / v$ ( $y_{r}$ is a characteristic roughness height), is shown in Figure 1 for a number of different types of rough surfaces. It can be seen that there is a large degree of uncertainty particularly with increased roughness. This dependence of $\mathrm{E}$ on the roughness of building materials has recently been 
the subject of study by Hammond and his co-workers. He peports ${ }^{10}$ that for conorete block appropriate values for $E_{\text {and }} P_{j}$ would be 0.568 and +2.83 ; and for general finish brick 0.064 and +6.55 respectively. For smooth walls we have taken $E$ to be 9 and $P_{j}$ to be -2.77 where $P_{j}$ has been calculated from the empirical correlation ${ }^{*}$

$P_{j}=9.24\left(\left(\frac{\mathrm{Pr}}{\mathrm{Pr}_{t}}\right)^{3 / 4}-1\right)\left(1+0.28 \exp \left(-.007 \frac{\mathrm{Pr}}{\mathrm{Pr}_{t}}\right)\right)$

where $\mathrm{Pr}$ is the molecular Prandtl number, taken to be 0.7 for air and the turbulent Prandtl number Prt is taken to be unity. The effects of variations to these parameters on the predicted flow field of a tunnel fire are examined in the following section.

\section{APPLICATION TO TUNNEL FIRE PROBLEM}

In the first Symposium ${ }^{1}$ comparisons between predictions and experimental measurements were reported for full scale fires conducted in the disused zwenberg railway tunnel in Austria. These experiments are used again for comparison with the model JASMINE, improved by the incorporation of the six flux radiation model and the use of rough wall, wall functions. The schematic of the tunnel is shown in Eig 2. It is 390 $m$ long and has a $2.18 \%$ gradient from the south to the north portal. It had been sealed at the south portal with a fan inserted in the end wall to provide longitudinal ventilation. The fire was situated $108 \mathrm{~m}$ from the south portal. The experiments conducted and the steady state and time dependent numerical predictions are described in detail in Ref 3 .

Two of the experiments chosen for this study have an imposed longitudinal ventilation of $2 \mathrm{~m} / \mathrm{s}$ and $4 \mathrm{~m} / \mathrm{s}$. Both involved fires of 200 litres of petroleum fuel burnt in a $2.6 \mathrm{~m}$ square tray. For the two ventilation rates, the heat outputs were estimated to be $20.25 \mathrm{MW}$ and $24.95 \mathrm{MW}$ respectively. Gas temperature, and concentrations of $\mathrm{CO}_{2}$ and $\mathrm{O}_{2}$ were measured at the seven measurement statiols shown in the Figure. For brevity, only stations 1 and 2 will be used for comparison with the predictions.

The numerical representation of the tunnel and fire source have been described in detail in Ref. 3 and will not be repeated here except to recall that smooth wall functions were used to account for momentum transfer to the walls. A lumped heat transfer coefficient was used which varied between 5 and $40 \mathrm{~W} / \mathrm{m}^{2} \mathrm{~K}$ depending on the near wall gas temperature. Since the data only provided maximum gas temperatures and gas concentrations, predictions for the steady state simulation only are shown here. Transient calculations are reported in Ref.3.

The predictions have been made assuming uniform radiative properties of the walls and combustion products. The wall (brick) emissivity has been taken to be 0.93 and the absorption and scattering coefficients of the combustion products everywhere taken as 1.0 and 0.01 respectively, there being no suitable measurements available to guide this choice.

\section{RESULTS AND DISCUSSION}

The various treatments have only a very slight effect on the predictions of carbon dioxide and oxygen concentrations and are therefore not included here. Results of the predictions for temperature and comparison with the measurements are summarised in Fig 3 . It shows 


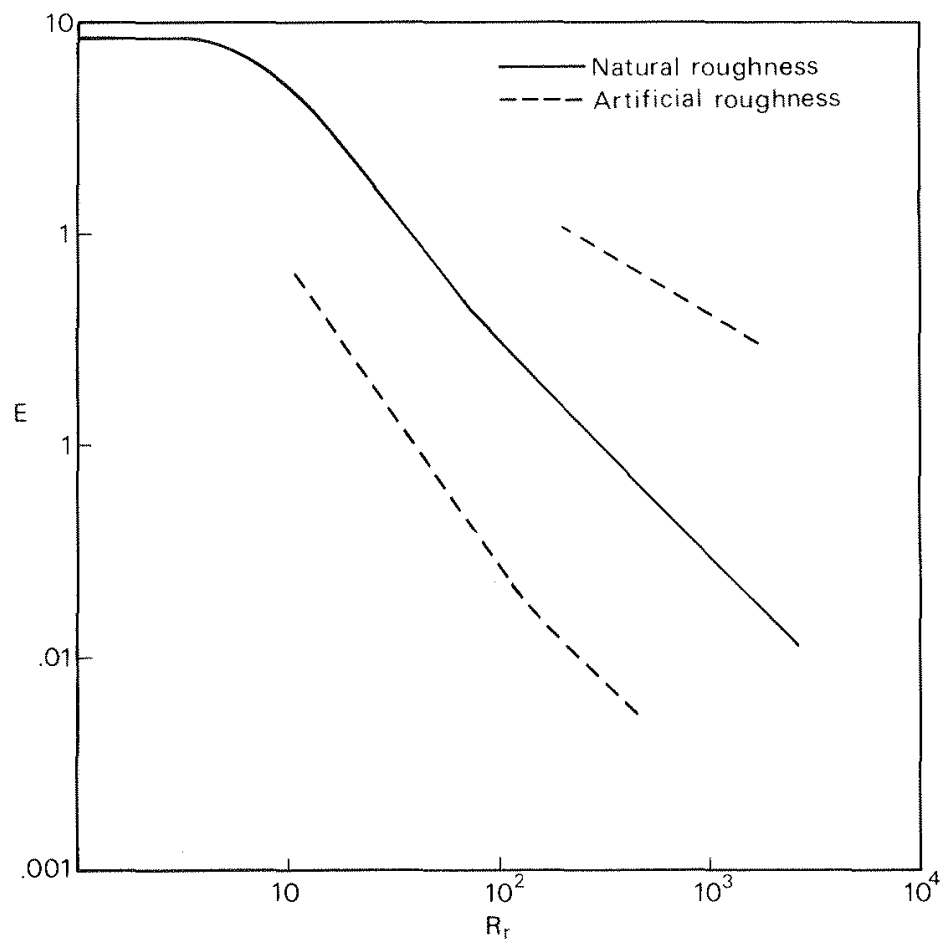

Figure 1 Summary of measured variation of roughness parameter $E$ with roughness Reynolds number $\mathrm{R}_{r}$ (See ref 9 )

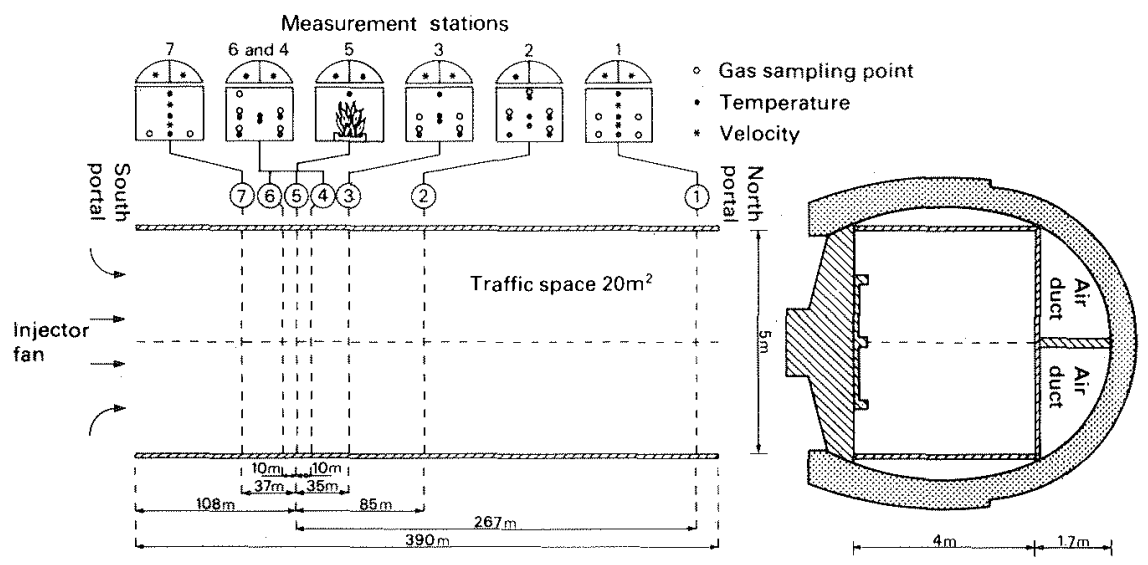

Figure 2 Schematic plan and cross section of tunnel 

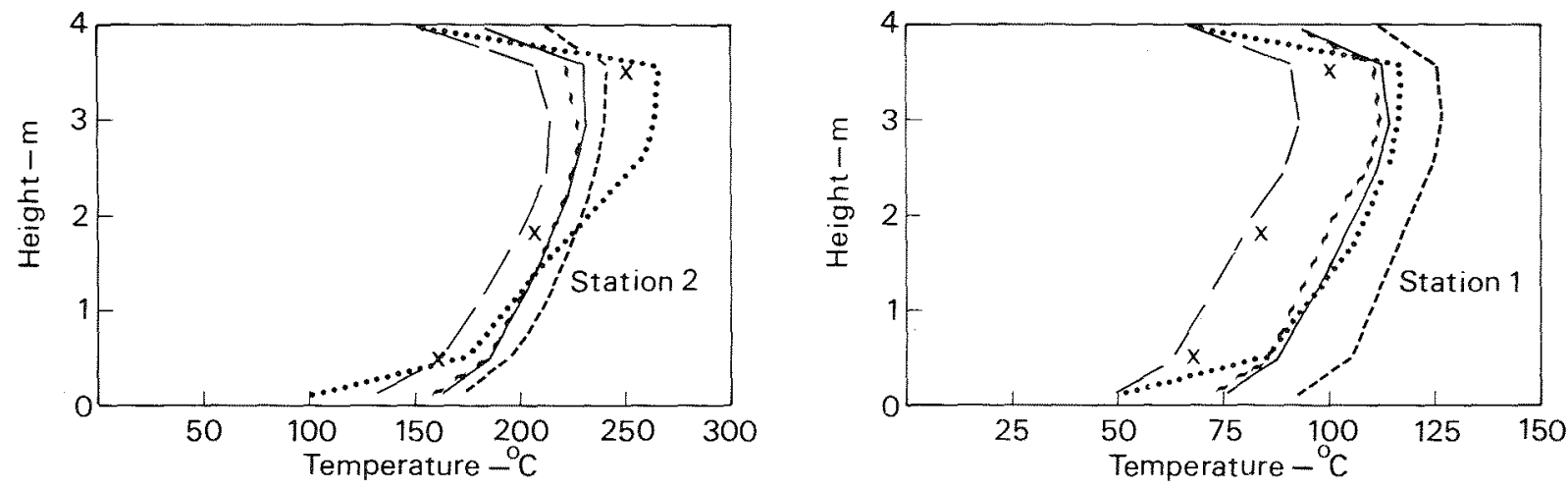

(a) Ventilation rate $2 \mathrm{~m} / \mathrm{s}$

号
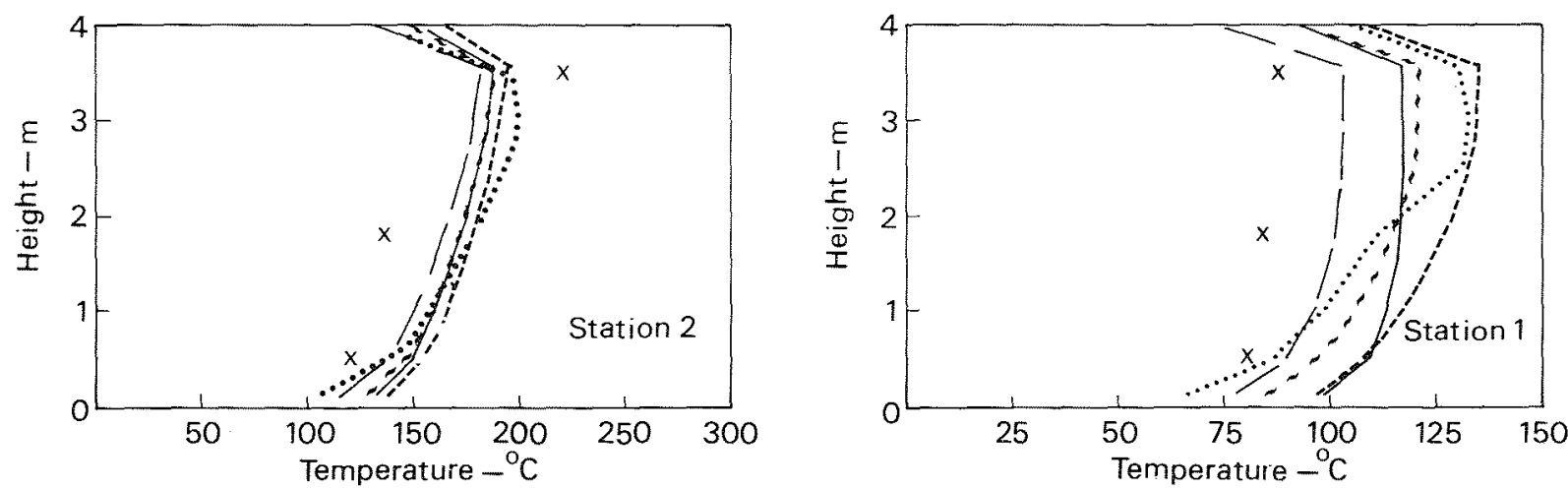

(b) Ventilation rate $4 \mathrm{~m} / \mathrm{s}$

Figure 3 Effect on temperature predictions of wall roughness and radiation Measurements $x$ : Predictions see table 1 
measurements and predictions of vertical profiles at measurement stations 1 and 2 for both ventilation rates.

TABLE 1. Key to symbols used in Figure 3

$\begin{array}{cccc}E & \mathrm{P}_{j} & \text { Radiation Treatment } & \text { Symbol } \\ 9.0 & -2.77 & \text { heat transfer coefficient } & \ldots \ldots \ldots \\ 9.0 & -2.77 & \text { six flux } & \ldots \\ 0.568 & -2.77 & \text { six flux } & \ldots \\ 0.064 & -2.77 & \text { six flux } & \ldots \\ 0.064 & +6.56 & \text { six flux }\end{array}$

The modifications do have an increasingly significant effect on the temperature predictions as the accumulated heat loss from the combustion products becomes apparent the further they move downstream from the fire. The six-flux and lumped heat transfer coefficient predictions using smooth wall functions produce, broadly, the same maximum temperatures but the six-flux model in general tends to 'smear' the profiles due to the exchange of radiant heat not just with the walls but also with neighbouring gas cells.

Variation of $\mathrm{E}$, as suggested by the work of Hammond produces a large effect at the exit measurement station(1) reducing peak temperatures there by around $30^{\circ} \mathrm{C}$ in both ventilation conditions although this is reduced somewhat if a parallel increase in $P_{j}$ is included.

A plot of convective heat transfer coefficient at the ceiling, for measurement station $2(85 \mathrm{~m}$ downstream from the fire) as a function of wall roughness is shown in Figure 4 for the two ventilation rates. For comparison, it is also displayed for the $2 \mathrm{~m} / \mathrm{s}$ case at the ceiling in the cold flow at measurement station 7 (37 $\mathrm{m}$ upstream from the fire). The smooth wall prediction compares favourably with 1 iterature values $(6.4$ $\left.W / m^{2} K\right)$.

A plot of radiative heat flux to the floor of the tunnel is shown in Figure 5. This is particularly important from the practical design point of view. A 'threshold of pain' line has been included, based"1 on a radiant flux of $2 \mathrm{~kW} / \mathrm{m}^{2}$, which clearly shows that the levels of unacceptable, life threatening radiant flux extend about $70 \mathrm{~m}$ downstream from the fire at $2 \mathrm{~m} / \mathrm{s}$ but only about $22 \mathrm{~m}$ downstream at $4 \mathrm{~m} / \mathrm{s}$.

\section{CONCLUSIONS}

The inclusion of the six-flux radiation model has improved the realism of the predictions of the 'far field' in this problem. It is not expected that this model will be adequate for the treatment of near-field effects where the discrete transfer method appears to offer significant advantages. Significant improvements should however be possible by linking local scattering and absorption coefficients to, for example, the product mass fraction used in the simple combustion models.

There is clearly more work required both on the characterisation of surface roughness for realistic building materials, and indeed for the surfaces often used in experimental fire test rigs, as well as further 


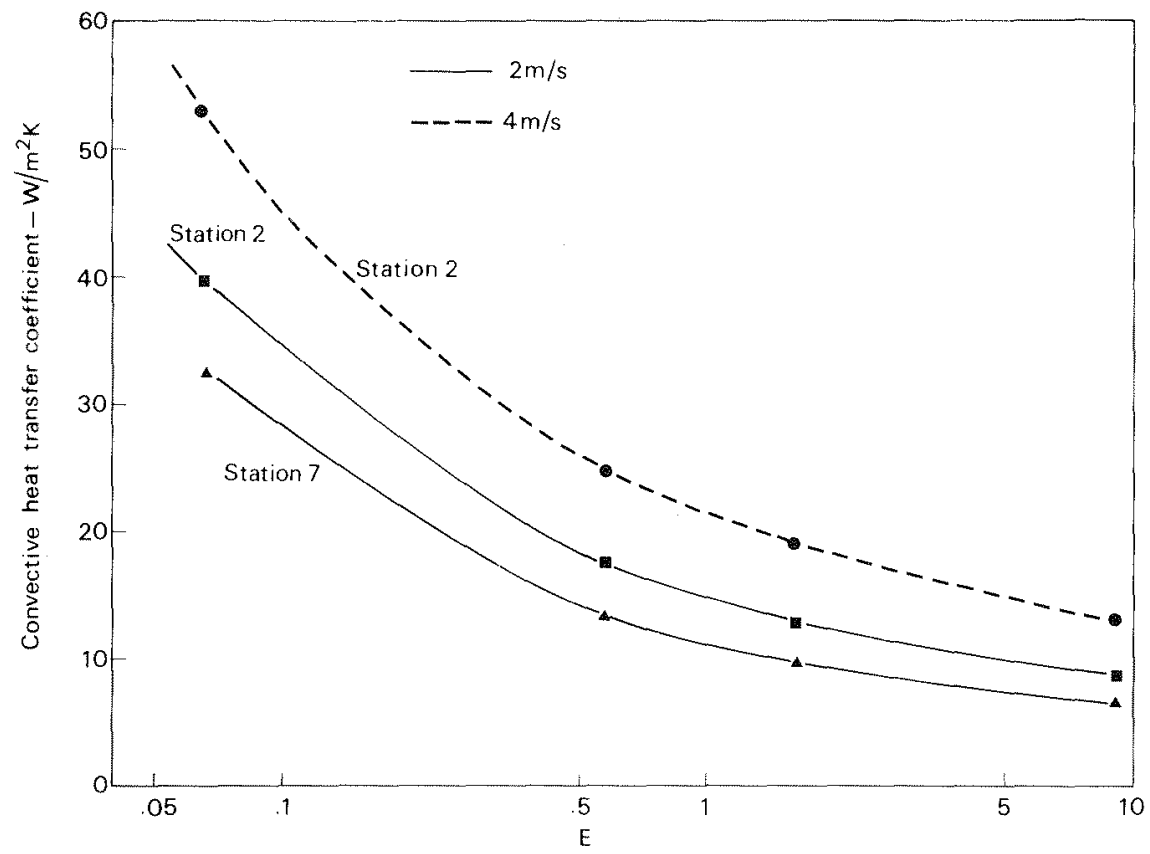

Figure 4 Convective heat transfer coefficient calculated from wall functions

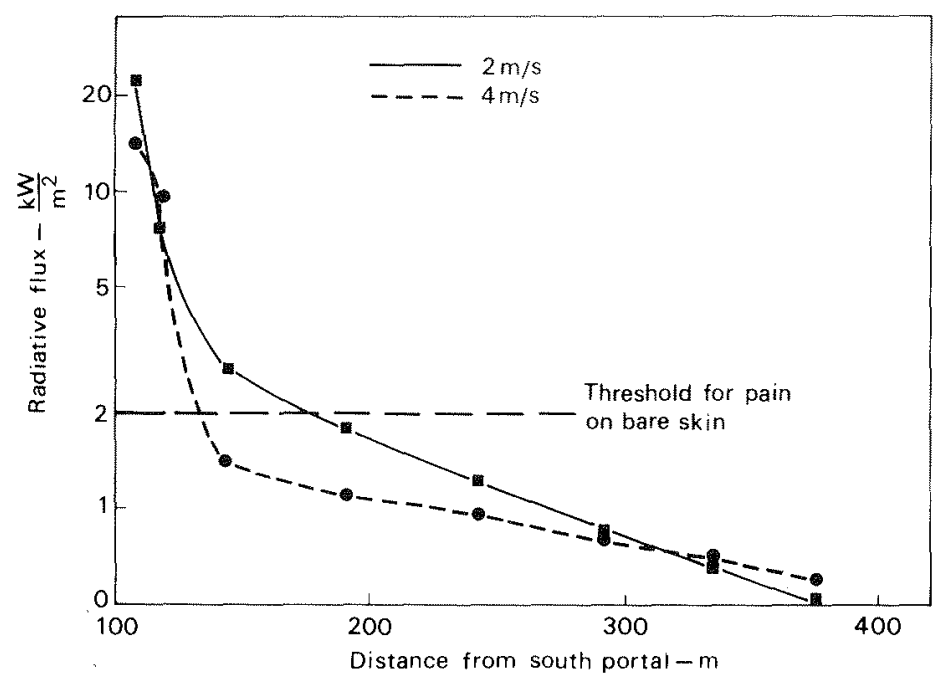

Figure 5 Radiative flux on floor of tunnel (Predicted by JASMINE) 
validation of the rough wall, wall function approach. The inclusion of these ireatments does, however, improve the universality of the field modelling approach that is a feature of its treatment of free flowing convection.

\section{ACKNOWLEDGEMENTS}

The authors are grateful to Geoff Hammond of the Applied Energy Group, School of Mechanical Engineering, Cranfield Institute of Technology for the use of his unpublished data on the roughness of building materials.

The paper forms part of the work of the Fire Research Station, Building Research Establishment, Department of the Enyironment, UK. It is contributed by permission of the Director, Building Research

Establishment. (c) Crown copyright 1987

\section{REFERENCES}

1. Cox G, Kumar $S$ and Markatos N C. Some field model validation studies, p 159 Proc First International Symposium on Fire Safety Science, Hemisphere, 1986.

2. Cox G, and Kumar $S$. The mathematical modeliing of fire in forced ventilated enclosures. p 692 in Proc 18th DOE Nuclear Airborne Waste Management Conference, Harvard University, 1984; see also Combus. Sei. Technol, 52, 7, 1987.

3. Kumar $S$ and $\operatorname{Cox} G$. Mathematical modelling of fire in road tunnels validation of JASMINE. Transport and Road Research Laboratory Contractor Report No 28, Crowthorne 1986.

4. Cox G. Combustion Science and Technology, 17, 75, 1977.

5. Charnley D. Radiating flamelet models of turbulent buoyant diffusion flames. PhD Thesis. Dept of Aeronautics and Astronautics, University of Southampton, 1986.

6. Viskanta $R$ and Menguc $M$ P. Radiation heat transfer in combustion systems. Prog Energy Comb Seience, 13, 97, 1987.

7. Shah N G. New method of computation of radiant heat transfer in combustion chambers. PhD Thesis. Dept of Mechanical Engineering, Imperial college, London 1979.

8. Schlichting H. Boundary Layer Theory. McGraw Hill, 1960.

9. Jayatilleke $\mathrm{C} L \mathrm{~L}$. The influence of Prandt 1 number and surface roughness on the resistance of the laminar sub layer to momentum and heat transfer. Prog in Heat and Mass Transfer, 1, 193, 1969.

10. Hammond G P. Private communication 1987.

11. Simms D L and Hinkley P L. Protective clothing against flames and heat. Joint Fire Research Organistion Fire Research Special Report No.3, 1960. 